\title{
Asociación entre cognición y depresión en adultos mayores panameños con cognición normal y deterioro cognitivo leve.
}

\author{
Ambar R. Pérez-Lao ${ }^{1,2, *}$, Diana C. Oviedo, ${ }^{1,2}$, Gabrielle B. \\ Britton $^{1,2}$ \\ ${ }^{1}$ Escuela de Psicología, Universidad Católica Santa María la Antigua 0819-08550 \\ ${ }^{2}$ Centro de Neurociencias y Unidad de Investigación Clínica, Instituto de Investigaciones Científicas \\ y Servicios de Alta Tecnología (INDICASAT AIP) 0843-01103, Pmá 5
}

*Autor para Correspondencia. E-mail: arperla23@gmail.com

Recibido: 11 de mayo de 2020

Aceptado: 10 de junio de 2020

\begin{abstract}
Resumen
La depresión y el deterioro cognitivo leve (DCL) en el adulto mayor están relacionados con el desarrollo de distintos tipos de demencia y disminución en la funcionalidad. Se realizó un estudio descriptivo de corte transversal con una muestra de 73 adultos mayores panameños de 65 años o más, de la cohorte del Panama Aging Research Initiative (PARI). Se midió estado de salud y funcionalidad en las actividades de la vida diaria. El funcionamiento cognitivo fue medido con pruebas neuropsicológicas cuyos puntajes se combinaron para formar seis dominios: cognición global, memoria, lenguaje, habilidades visuoespaciales, atención y funciones ejecutivas. Para medir síntomas depresivos se utilizó la Escala de Depresión Geriátrica (GDS-30). Se realizó un análisis de covarianza (ANCOVA) comparando sujetos con y sin posible depresión para cada dominio cognitivo, en sujetos con DCL y cognición normal $(\mathrm{CN})$ y controlando por las variables de edad y escolaridad. Se encontraron diferencias significativas entre los grupos, el grupo con posible depresión y DCL tenía menores puntuaciones en comparación con los otros grupos en cognición global. Se realizaron dos análisis de regresión lineal para determinar los factores asociados con el desempeño cognitivo en cada grupo diagnóstico individualmente. En el grupo $\mathrm{CN}$ la escolaridad fue un predictor significativo del desempeño en los dominios cognitivos, mientras que en el DCL se encontró que la edad, la educación, la depresión y el índice de masa corporal (IMC) estaban relacionados con menor desempeño cognitivo. En el grupo de DCL, depresión predijo bajo desempeño en los dominios cognitivos de funciones ejecutivas y atención. Estos resultados podrían aportar al desarrollo de políticas de salud dirigidas a adultos mayores, y a esfuerzos especializados de prevención e intervención enfocados en minimizar la discapacidad y sus mediadores.
\end{abstract}

Palabras clave: depresión, envejecimiento, cognición, Alzheimer, funciones cognitivas. 


\begin{abstract}
Depression and mild cognitive impairment (MCI) in elderly adults are associated with the development of different types of dementia and the reduction of functionality. The following is a descriptive study analyzing a transversal sample of 73 Panamanian elderly adults of 65 or more years of the Panama Aging Research Initiative (PARI) cohort. We measured health status and functionality in activities of daily living. Cognitive function was measured with neuropsychological tests. Scores were combined in six cognitive domains: global cognition, memory, language, visuospatial abilities, attention and executive function. To measure symptoms of depression, the 30item Geriatric Depression Scale (GDS-30) was used. A covariance analysis (ANCOVA) was performed between participants with and without possible depression on both groups of normal cognition $(\mathrm{CN})$ and $\mathrm{MCI}$, to measure cognitive performance. Participants in the group with possible depression and MCI, showed to have lower scores on global cognition that the other groups. Two linear regression analysis were performed for each group. In the group of normal cognition, education was the significant predictive factor on cognitive performance, while in the MCI group other factors like age, education, depression and body mass index were related to lower cognitive performance. In the MCI group depression predicted lower performance in executive functions and attention. These results could aid in the development of public health politics directed to elderly adults, and the efforts made in prevention and intervention, focused on minimizing disability and its mediators.
\end{abstract}

Keywords: depression, aging, cognition, Alzheimer, cognitive functions.

\title{
1. Introducción
}

Según el informe publicado por las Naciones Unidas en el 2019, la población mundial con mayor crecimiento es la de adultos mayores de 65 años. En el año 2019 se estimó que esta población abarca 9\% a nivel mundial, y se estima que para el año 2050 aumente a 16\% (1). Latinoamérica se encuentra entre las regiones en donde se proyecta una duplicación de la población de adultos mayores para el año 2050. En Panamá en el año 2019 el porcentaje de adultos mayores de 65 años era de aproximadamente $8.6 \%$ y se estipula que para el año 2050 será de $18.2 \%$ de la población total (2). La expectativa de vida se ha visto en aumento en Panamá ocupando el número 45 en el ranking de expectativa de vida mundial con 77.7 años de edad (2). Sin embargo, el incremento de la expectativa de vida no necesariamente es un indicador de que el envejecimiento sea sano y activo. Una mayor longevidad a menudo está acompañada de un aumento de los problemas de salud y deterioro funcional en el adulto mayor.

Entre las condiciones que más afectan la funcionalidad de los adultos mayores se encuentran la depresión y la demencia. La demencia afecta aproximadamente a un 5\% de la población mundial, mientras que la depresión afecta al 7\% (3). La depresión es un trastorno mental caracterizado por síntomas como ánimo deprimido o pérdida de intereses, cambios en el peso, insomnio o hipersomnia, fatiga o pérdida de energía, sentimiento de inutilidad y culpabilidad e incluso ideación suicida (4). La depresión es común en el adulto mayor y puede ser causada por factores biológicos como accidentes cerebrovasculares, y psicosociales como la pérdida de un ser querido (5). La depresión también se encuentra relacionada con el desarrollo de la demencia, incluyendo el tipo 
Alzheimer (6). La demencia de tipo Alzheimer (EA) es una enfermedad neurodegenerativa que causa declive en la memoria y otras habilidades de pensamiento con repercusiones en la capacidad para realizar las actividades de la vida diaria (7).

La depresión y EA comparten cambios neurológicos similares, entre ellos la pérdida neuronal hipocampal (8) y cambios en la materia blanca en regiones frontal, parietal o temporal (9). También comparten factores de riesgo como la expresión de Apolipoproteína $\varepsilon 4$, que es uno de los mayores factores de riesgo genético establecidos en la EA esporádica (10).

La depresión también se asocia con la etapa prodrómica de la EA conocida como deterioro cognitivo leve (DCL). Se ha observado en otros estudios que el porcentaje de personas con DCL y síntomas depresivos puede llegar a ser de $25 \%$ en muestras basadas en la comunidad y $40 \%$ en muestras clínicas (11). Incluso, en un meta-análisis reciente (6), se concluyó que los sujetos con DCL y síntomas depresivos tenían más probabilidades de progresar a una demencia, en especial de tipo Alzheimer. La depresión y el DCL también comparten factores de riesgo que son similares en la EA como ser del género femenino, más de 75 años, no tener pareja ni trabajo, pensionado o con bajo nivel de estudios (12).

Adultos mayores con depresión y DCL simultáneamente poseen mayor deterioro en la cognición en comparación con personas que solo padecen DCL. En individuos con ambas patologías existe un pobre desempeño en la atención, habilidades visuoespaciales y funciones ejecutivas, así como también distintos tipos de memoria como inmediata, semántica y a largo plazo (13)(14). En Panamá, se han reportado factores asociados a la coocurrencia de depresión y DCL como tener mas de 80 años, menor nivel de educación, mayor cantidad de enfermedades crónicas y problemas para desempeñar las actividades básicas de la vida diaria (ABVD) (15). El principal objetivo de esta investigación fue caracterizar los factores asociados a la depresión y cognición en una muestra de adultos mayores panameños con cognición normal o deterioro cognitivo leve.

\section{Materiales y Métodos}

\subsection{Diseño de estudio}

Se realizó una investigación descriptiva de tipo correlacional con enfoque cuantitativo y finalidad aplicada. Los análisis realizados fueron de tipo transversal y se utilizaron métodos estadísticos multivariantes.

\subsection{Muestra}

Los participantes formaban parte de la cohorte del Panama Aging Research Initiative (PARI), reclutados de la consulta externa del Servicio de Geriatría de la Caja del Seguro Social (15)(16). Los criterios de inclusión eran ser mayor de 65 años, poder leer y escribir, otorgar consentimiento informado al momento de la inclusión y estar disponibles para las visitas. Los criterios de exclusión abarcaban no cumplir con los requisitos de la edad o no haber asistido a la primera visita del estudio. No se incluyeron sujetos que estaban participando en otros estudios o en ensayos clínicos ni con alguna discapacidad física que les impidieran asistir a las visitas. 
La muestra fue no probabilística con muestreo por conveniencia. Estuvo constituida por 73 sujetos que fueron divididos en dos grupos: cognición normal $(\mathrm{CN} ; \mathrm{n}=41)$ y deterioro cognitivo leve (DCL, $\mathrm{n}=32$ ). El diagnóstico de individuos para la asignación de los grupos fue realizado por un equipo multidisciplinario de profesionales de la salud. Para el diagnóstico se evaluaron los cuestionarios y pruebas cognitivas. Se utilizó la Escala de Deterioro Global (GDS) (17), que divide el proceso de deterioro cognitivo en siete etapas: sin deterioro, deterioro cognitivo muy leve, deterioro cognitivo leve, deterioro cognitivo moderado (demencia leve), deterioro cognitivo moderado severo (demencia moderada), deterioro cognitivo severo (demencia moderada severa) y deterioro cognitivo muy severo (demencia severa).

Los participantes del grupo de cognición normal tenían una puntuación total de 28 o más en el Mini Examen del Estado Mental (MMSE) (18) y una puntuación de dos o menos en el GDS. El diagnóstico del grupo DCL se basó en los criterios clínicos previamente establecidos (19) y una puntuación entre 24 y 27 en el MMSE y de tres en el GDS. De acuerdo a criterios diagnósticos de DCL y CN, los participantes no presentaban alteraciones para realizar las actividades de la vida diaria.

\subsection{Instrumentos utilizados}

\subsubsection{Cuestionarios}

Se utilizaron dos cuestionarios para medir independencia en las actividades de la vida diaria. El Cuestionario de Actividad Funcional (FAQ) (20) consiste de 10 preguntas que determinan el nivel de dependencia en las actividades básicas e instrumentales de la vida diaria. Para cada pregunta se puntúa del cero al tres, y un mayor puntaje indica mayor dependencia.

El Cuestionario de Actividades Instrumentales de la Vida Diaria de Lawton and Brody (AIVD) (21) valora la capacidad funcional en las AIVD del participante en 10 dominios específicos: capacidad para utilizar el teléfono, hacer compras, preparación de la comida, cuidado de la casa, lavado de la ropa, cuidado personal, desplazamiento personal, uso de los medios de transporte, responsabilidad respecto a la medicación y administración de su economía. Los ítems se puntúan con uno o cero, con cero indicando si el participante es dependiente y " 1 " si es independiente.

Para evaluar la presencia de síntomas depresivos se utilizó la Escala de Depresión Geriátrica (GDS30) (22) que mide la presencia de síntomas depresivos en adultos mayores mediante 30 preguntas, que se puntúan 0 o 1 . Se califica de la siguiente manera: de cero a 10 es una puntuación normal, sin signos de depresión, y de 11 a 30 es presencia de posible depresión.

\subsubsection{Pruebas neuropsicológicas}

Se utilizaron distintas pruebas neuropsicológicas para medir el funcionamiento de los participantes. Se dividieron las pruebas en seis dominios cognitivos: cognición global, lenguaje, habilidades visuoespaciales, memoria, atención y funciones ejecutivas. Para medir la cognición global se utilizó el Mini Examen del Estado Mental (MMSE) compuesto por 30 ítems que incluye medidas en las áreas 
de orientación, lenguaje, aprendizaje, memoria y habilidades visuoespaciales. La puntuación máxima es de 30.

Para el dominio de lenguaje se utilizó el Test de Vocabulario de Boston (TVB) de 30 ítems y una prueba de fluidez verbal semántica. En el TVB se presentan 30 láminas con diferentes dibujos que el participante debe nombrar. En la prueba de fluidez semántica el participante nombra la mayor cantidad de animales en un minuto de tiempo, y se le otorga un punto por cada respuesta correcta. No se tomaron en cuenta las perseveraciones e intrusiones en la puntuación final.

Para medir habilidades visuoespaciales se utilizó la prueba de Figuras Solapadas de Poppelreuter que consiste en que el participante debe identificar varios objetos sobrepuestos en dos figuras. Además, se utilizó la prueba del reloj a la orden y a la copia. En la prueba a la orden se le indica al participante que dibuje un reloj a la orden sin modelo, y en la copia se le indica que reproduzca el dibujo de un reloj plasmado en una hoja. Se puntúan tres aspectos: esfera, manecillas y número. La puntuación máxima es de 10 puntos.

Se utilizó la prueba de Lista de 10 palabras (23) para medir memoria verbal. La aplicación de esta prueba consiste en leerle al participante una lista de 10 palabras que el participante debe recordar, se repite la lista de palabras un total de tres veces, y se suman las palabras correctas en cada ensayo. Después de 20 minutos se le indica al participante que diga todas las palabras que recuerde.

En el dominio de atención se utilizaron las pruebas de dígitos y el Trail Making Test (TMT), la parte A. La prueba de dígitos pertenece a la Escala de Memoria de Wechsler (WMS)(24). En la prueba de dígitos directos el evaluador dicta una secuencia de números, y al terminar cada serie, el participante debe repetir las secuencias. La prueba consiste en nueve series con dos intentos cada una, con dificultad creciente. En la prueba de Trail Making Test parte A (25), el participante debe trazar una línea continua uniendo los números del 1 al 20 en orden sin levantar el lápiz del papel. La calificación es en base al tiempo que le toma al participante culminar la prueba.

El dominio de funciones ejecutivas fue medida con dígitos inversos de la WMS (24), el TMT parte B (25) y la prueba del reloj a la orden (26). En la subprueba de dígitos, el participante debe repetir los números que el evaluador dicta de forma inversa. Consta de nueve secuencias todas con dos ensayos, y se puntúa dos puntos si el participante logra culminar los dos ensayos, un punto si realiza solo uno y cero si no realiza ninguno. El TMTB incluye números del uno al ocho y las letras de la "A" a la "G", que el participante debe unir de manera alterna lo más rápido posible. Para la calificación se toma en cuenta el tiempo. En la prueba del reloj, el participante debe dibujar un reloj completo a la orden sin modelo, se califica tomando en cuenta esferas, manecillas y números con una puntuación máxima de 10 puntos en total.

\subsubsection{Consideraciones Éticas}

El diseño e implementación del estudio van de acorde con la Guía Tripartita Armonizada para la Buenas Prácticas Clínicas de la International Conference on Harmonization ICH, con las regulaciones locales vigentes de acuerdo con los principios éticos establecidos en la declaración de Helsinki, versión 2008. Este estudio se ha llevado a cabo con la guía de las consideraciones éticas establecidas en el Código de Buenas Prácticas Éticas de Investigación de la USMA. El protocolo del 
estudio y todos los procedimientos que se realizaron dentro del mismo, fueron aprobados por el Comité de Bioética del Instituto Conmemorativo Gorgas de Estudios de la Salud y de la Caja del Seguro Social.

\subsubsection{Análisis de resultados}

Para realizar la división de los dominios cognitivos y compararlos entre los grupos diagnósticos, las puntuaciones directas de las pruebas neuropsicológicas se convirtieron en puntuaciones estándar (z). Se invirtieron los valores $z$ en los casos que fuese necesario para que un valor de $z$ positivo indicara mejor rendimiento para todas las pruebas. Posteriormente, se calculó el promedio de las puntuaciones z de pruebas específicas para generar seis dominios cognitivos: cognición global (MMSE), lenguaje (Test reloj copia y Poppelreuter), memoria (Lista de 10 palabras a corto y largo plazo), atención (Dígitos directos y TMTA) y funciones ejecutivas (TMTB, reloj a la orden y dígitos inversos).

Primero se realizó un análisis de modelo lineal ANOVA univariable para las variables cuantitativas y chi cuadrado $(\chi 2)$ para las variables cualitativas (ver Tabla 1). Se realizó un análisis de covarianza (ANCOVA) entre los grupos de DCL y CN con y sin depresión. El punto de corte para determinar si los participantes tenían signos de depresión fue una puntuación de GDS-30 $\geq 10$ y sin depresión GDS-30<10. Las covariables fueron edad y escolaridad. Por último, se realizaron análisis de regresión lineal por separado en los grupos de CN y DCL para evaluar las variables edad, escolaridad, índice de masa corporal (IMC) y síntomas de depresión (GDS-30) como predictivas del desempeño en los dominios cognitivos. Se reportan los valores de Beta (b) y error estándar de beta. Los análisis estadísticos se realizaron con el programa SPSS versión 20.0. El nivel de significancia se estableció en $p<0.05$.

\section{Resultados}

En la tabla 1 se resumen los datos sociodemográficos. Dentro de la muestra de 73 participantes, el sexo predominante fue el femenino con un $69.9 \%$ del total de la muestra. No se encontraron diferencias significativas entre grupos en edad, sexo, escolaridad o GDS-30.

Tabla 1

Comparación de datos sociodemográficos y cuestionarios entre CN y DCL

\begin{tabular}{llllll}
\hline Características & $\begin{array}{l}\mathrm{CN} \\
(\mathrm{n}=41) \\
\text { Media } \\
(\%)\end{array}$ & $\begin{array}{l}\text { DCL } \\
(\mathrm{n}=32) \\
\text { Media (DE) ó \# }\end{array}$ & $\begin{array}{l}\text { Fó } \chi^{2} \\
(\%)\end{array}$ & $p$ \\
\hline Escolaridad & $9.95(3.84)$ & $8.53(3.42)$ & 2.70 & 0.10 \\
$\%$ Mujeres & $31(75.6 \%)$ & $20(62.5 \%)$ & 1.47 & 0.30 \\
Edad & $74.87(5.59)$ & $77.52(7.74)$ & 2.90 & 0.09 \\
IMC & $26.29(4.85)$ & $24.24(4.28)$ & 3.38 & 0.70
\end{tabular}




$\begin{array}{lllll}\text { FAQ } & 1.24(2.7) & 2.13(2.68) & 1.92 & 0.17 \\ \text { IF } & 0.92(0.16) & 0.92(0.11) & 0.00 & 0.96 \\ \text { ABVD+AIVD } & 9.10(1.62) & 9.09(1.12) & 0.00 & 0.99 \\ \text { \%GDS-30>10 } & 9(22.0 \%) & 8(25.0 \%) & 0.09 & 0.76 \\ \text { GDS-30 } & 5.59(4.97) & 7.59(5.25) & 2.79 & 0.09\end{array}$

Nota. $\mathrm{CN}=$ cognición normal; DCL=deterioro cognitivo leve; IMC=Índice de masa corporal; $\mathrm{FAQ}=$ cuestionario de la actividad funcional; $\mathrm{ABVD}=$ actividades básicas de la vida diaria; $\mathrm{AIVD}=$ actividades instrumentales de la vida diaria; GDS-30=escala de depresión geriátrica (30 ítems); IF=índice de funcionalidad.

Se realizó un análisis comparando el desempeño en los dominios cognitivos entre los grupos de deterioro cognitivo leve (DCL) y cognición normal $(\mathrm{CN})$. En la tabla 2 se resume la comparación entre los grupos diagnósticos, donde las diferencias fueron significativas en los dominios de cognición global $[F(72)=5.96, p=0.02]$ y habilidades visuoespaciales $[F(72)=13.80, p<0.001]$. No se observaron diferencias significativas entre los dominios de lenguaje $[F(72)=.96, p=0.33$ ], memoria $[F(72)=.14, p=0.71]$, funciones ejecutivas $[F(72)=1.52, p=0.22]$ o atención $[F(72)=.23$, $p=0.63]$. Sin embargo, cabe mencionar que las puntuaciones del grupo DCL en comparación con el grupo $\mathrm{CN}$ fueron menores en cada dominio.

\section{Tabla 2}

Comparación entre CN y DCL en dominios cognitivo

\begin{tabular}{lllll}
\hline $\begin{array}{l}\text { Dominio } \\
\text { cognitivo }\end{array}$ & $\begin{array}{l}\mathrm{CN} \\
\mathrm{n}=41 \\
\text { Media }(\mathrm{DE})\end{array}$ & $\begin{array}{l}\mathrm{DCL} \\
\mathrm{n}=32 \\
\text { Media }(\mathrm{DE})\end{array}$ & $F$ & $p$ \\
\hline Cognición global & $0.51(0.35)$ & $0.20(0.51)$ & 5.96 & 0.02 \\
Lenguaje & $0.42(0.52)$ & $0.05(0.66)$ & 0.96 & 0.33 \\
$\begin{array}{l}\text { Habilidades } \\
\text { Visuoespaciales }\end{array}$ & $0.38(0.25)$ & $0.13(0.52)$ & 13.80 & 0.00 \\
Memoria & $0.66(0.59)$ & $-0.26(0.55)$ & 0.14 & 0.71 \\
$\begin{array}{l}\text { Funciones } \\
\text { Ejecutivas }\end{array}$ & $0.35(0.60)$ & $-0.25(0.73)$ & 1.52 & 0.22 \\
$\begin{array}{l}\text { Atención } \\
\text { Nota. CN=cognición normal; DCL=Deterioro Cognitivo Leve. }\end{array}$ & & \\
\end{tabular}


En la tabla 3 se observa la comparación entre participantes CN y DCL con y sin posible depresión. Se encontraron diferencias significativas en el dominio cognitivo de cognición global $[\mathrm{F}(1,69)=6.66$, $p<0.01]$. No se encontraron diferencias significativas en los dominios restantes.

\section{Tabla 3}

Comparación de sujetos con y sin posible depresión entre los grupos CN y DCL

\begin{tabular}{|c|c|c|c|c|c|c|}
\hline & $\begin{array}{l}\mathrm{CN} \\
\mathrm{n}=41\end{array}$ & & $\begin{array}{l}\text { DCL } \\
\mathrm{n}=32 \\
\end{array}$ & & & \\
\hline $\begin{array}{l}\text { Dominio } \\
\text { Cognitivo }\end{array}$ & $\begin{array}{l}\text { Sin } \\
\text { depresión } \\
\mathrm{n}=32 \\
\text { Media (DE) }\end{array}$ & $\begin{array}{l}\text { Con posible } \\
\text { depresión } \\
\mathrm{n}=9 \\
\text { Media (DE) }\end{array}$ & $\begin{array}{l}\text { Sin } \\
\text { depresión } \\
\mathrm{n}=24 \\
\text { Media (DE) }\end{array}$ & $\begin{array}{l}\text { Con posible } \\
\text { depresión } \\
\mathrm{n}=8 \\
\text { Media (DE) }\end{array}$ & $F$ & $p$ \\
\hline $\begin{array}{l}\text { Cognición } \\
\text { Global }\end{array}$ & $0.38(0.09)$ & $0.61(0.16)$ & $0.17(0.10)$ & $-0.30(0.17)$ & 6.66 & 0.01 \\
\hline Lenguaje & $0.39(0.10)$ & $0.13(0.19)$ & $-0.09(0.12)$ & $0.09(0.21)$ & 1.78 & 0.19 \\
\hline $\begin{array}{l}\text { Habilidades } \\
\text { visuoespaciales }\end{array}$ & $0.32(0.07)$ & $0.30(0.13)$ & $0.00(0.08)$ & $0.24(0.14)$ & 1.33 & 0.25 \\
\hline Memoria & $0.53(0.11)$ & $0.77(0.20)$ & $-0.37(0.12)$ & $-0.40(0.22)$ & 0.61 & 0.44 \\
\hline Atención & $0.05(0.07)$ & $0.20(0.13)$ & $0.09(0.08)$ & $-0.07(0.14)$ & 1.88 & 0.17 \\
\hline $\begin{array}{l}\text { Funciones } \\
\text { ejecutivas }\end{array}$ & $0.18(0.06)$ & $0.41(0.12)$ & $0.02(0.07)$ & $0.02(0.13)$ & 1.17 & 0.28 \\
\hline
\end{tabular}

Nota. $\mathrm{CN}=$ cognición normal; DCL= deterioro cognitivo leve.

En la tabla 4 se presenta la regresión lineal del grupo CN. En el grupo $\mathrm{CN}$ la variable de escolaridad predijo desempeño en los dominios cognitivos de lenguaje $(p=0.00)$, atención $(p=0.01)$ y habilidades visuoespaciales $(p=0.048)$. Las otras variables de edad, IMC y GDS-30 no tuvieron asociación con ningunos de los dominios cognitivos medidos.

\section{Tabla 4}

Regresión lineal del grupo $C N$

\begin{tabular}{lllll}
\hline $\begin{array}{l}\text { Dominio } \\
\text { Cognitivo }\end{array}$ & $\begin{array}{l}\text { Escolaridad } \\
\mathrm{B}(\mathrm{EE})\end{array}$ & $\begin{array}{l}\text { Edad } \\
\mathrm{B}(\mathrm{EE})\end{array}$ & $\begin{array}{l}\text { IMC } \\
\mathrm{B}(\mathrm{EE})\end{array}$ & $\begin{array}{l}\text { GDS-30 } \\
\mathrm{B}(\mathrm{EE})\end{array}$ \\
\hline $\begin{array}{l}\text { Cognición Global } \\
\text { Lenguaje }\end{array}$ & $0.01(0.01)$ & $-0.01(0.01)$ & $0.01(0.01)$ & $-0.00(0.01)$ \\
$\begin{array}{l}\text { Habilidades } \\
\text { visuoespaciales }\end{array}$ & $0.05(0.02)^{* * *}$ & $0.01(0.01)$ & $0.01(0.01)$ & $0.02(0.01)$ \\
& $0.02(0.01)^{*}$ & $-0.00(0.01)$ & $0.00(0.01)$ & $0.00(0.01)$
\end{tabular}


Memoria

$0.03(0.02)$

$-0.03(0.02)$

$-0.00(0.02)$

$0.00(0.02)$

Funciones

Ejecutivas

$0.04(0.02)$

$-0.00(0.02)$

$0.01(0.02)$

$-0.02(0.02)$

Atención

$0.05(0.02)^{* *}$

$-0.01(0.01)$

$-0.00(0.02)$

$-0.01(0.02)$

Nota. $\mathrm{CN}=$ cognición normal; $\mathrm{DCL}=$ deterioro cognitivo leve; IMC=índice de masa corporal; GDS$30=$ escala de depresión geriátrica (30 items)

${ }^{* *} p<<0.001 .{ }^{* *} p<0.01 .{ }^{*} p<0.05$.

En cuanto al análisis de regresión lineal del grupo DCL, se observa en la tabla 5 que en atención se encontró correlación con las variables predictivas de edad ( $p=0.023)$, IMC $(p=0.015)$ y GDS-30 $(p=0.005)$, y no se observó correlación con escolaridad. En funciones ejecutivas se encontró correlación con las variables de escolaridad $(p=0.040)$ y GDS-30 $(p=0.013)$, pero no hubo correlación con edad y IMC. En el dominio cognitivo de habilidades visuoespaciales se encontró correlación con la variable predictiva de escolaridad $(p=0.038)$, y no se encontró correlación con las variables de edad, IMC y GDS-30. En los dominios cognitivos de memoria, lenguaje y cognición global no se encontró correlación con las variables predictivas de escolaridad, edad, IMC y GDS-30.

\section{Tabla5}

Regresión lineal del grupo DCL

\begin{tabular}{|c|c|c|c|c|}
\hline $\begin{array}{l}\text { Dominio } \\
\text { Cognitivo }\end{array}$ & $\begin{array}{l}\text { Escolaridad } \\
\mathrm{B}(\mathrm{EE})\end{array}$ & $\begin{array}{l}\text { Edad } \\
\mathrm{B}(\mathrm{EE})\end{array}$ & $\begin{array}{l}\text { IMC } \\
\text { B (EE) }\end{array}$ & $\begin{array}{l}\text { GDS-30 } \\
\mathrm{B}(\mathrm{EE})\end{array}$ \\
\hline Cognición Global & $0.01(0.02)$ & $-0.00(0.01)$ & $0.01(0.02)$ & $-0.03(0.02)$ \\
\hline Lenguaje & $0.00(0.03)$ & $-0.02(0.02)$ & $-0.03(0.03)$ & $-0.04(0.02)$ \\
\hline $\begin{array}{l}\text { Habilidades } \\
\text { visuoespaciales }\end{array}$ & $0.05(0.02)^{* *}$ & $-0.01(0.01)$ & $-0.00(0.02)$ & $-0.01(0.02)$ \\
\hline Memoria & $0.01(0.02)$ & $-0.01(0.01)$ & $0.04(0.02)$ & $0.01(0.02)$ \\
\hline $\begin{array}{l}\text { Funciones } \\
\text { ejecutivas }\end{array}$ & $0.06(0.03)^{*}$ & $-0.01(0.01)$ & $-0.05(0.03)$ & $-0.06(0.02)^{*}$ \\
\hline Atención & $0.04(0.02)$ & $-0.03(0.01)^{*}$ & $-0.05(0.02)^{*}$ & $-0.05(0.02)^{* *}$ \\
\hline
\end{tabular}

Nota. $\mathrm{CN}=$ cognición normal; $\mathrm{DCL}=$ deterioro cognitivo leve; IMC=índice de masa corporal; GDS$30=$ escala de depresión geriátrica (30 items)

${ }^{* * *} \mathrm{p}<0.001 .{ }^{* *} \mathrm{p}<0.01 .{ }^{*} \mathrm{p}<0.05$.

\section{Discusión}

El objetivo general de este estudio fue determinar la asociación entre depresión y el desempeño en seis dominios cognitivos en una muestra de participantes con cognición normal y DCL. Acorde al criterio diagnóstico, los sujetos con DCL tuvieron menor desempeño en la mayoría de los dominios cognitivos, en especial en el dominio de cognición global y habilidades visuoespaciales. Las pruebas 
utilizadas para medir los dominios cognitivos son eficientes para el diagnóstico diferencial de los sujetos con y sin deterioro cognitivo. Por ejemplo, en cuanto a pruebas rápidas y de fácil aplicación, múltiples estudios y en la atención primaria (27) utilizan el MMSE para el diagnóstico preliminar de deterioro cognitivo y demencias (28), así como la prueba del reloj (29).

Los resultados de esta investigación concuerdan con otros estudios que muestran que la presencia de posible depresión en DCL afecta el desempeño cognitivo (11)(14). En comparación con los otros grupos, el subgrupo de sujetos DCL con posible depresión mostró un mayor declive en el dominio de cognición global. La combinación de depresión y DCL y su repercusión en el deterioro cognitivo global podría ser una señal del desarrollo de una enfermedad de Alzheimer (30) o un factor de riesgo para el desarrollo de la demencia (6)(31). No obstante, para poder resolver esta hipótesis sería necesario continuar el estudio de forma longitudinal, siguiendo a este subgrupo de sujetos con ambas patologías para observar si se desarrolla la EA en la combinación de DCL y depresión.

En este estudio, se observó que una mayor cantidad de síntomas depresivos en sujetos con DCL es un factor que afecta el desempeño en distinto dominios cognitivos como funciones ejecutivas y atención, así como otros estudios han reportado efectos sobre la memoria y funciones visuoespaciales (14)(32)(33). En contraste, una mayor cantidad de síntomas depresivos no se encontraron relacionados con menor desempeño cognitivo en adultos mayores con cognición normal. Este resultado podría indicar que la presencia de síntomas depresivos afecta más a individuos con DCL que aquellos con CN.

Entre las limitaciones de este estudio se encuentra el diseño transversal que no permite determinar causalidad entre depresión y cognición o su relación en cuanto a la progresión de DCL a otros estadios como la demencia de tipo EA. Otra limitación está en la definición de posible depresión en este estudio mediante el puntaje del GDS-30, se podrían agregar herramientas como el DSM-V o el Cuestionario de Neuropsiquiatría de Cunnings para hacer su diagnóstico más certero, sin embargo son herramientas poco factibles en investigación (6). Sin embargo, el GDS-30 es una de las más utilizadas dentro de la literatura para encontrar síntomas depresivos (34). En Panamá se encontró que la escala del GDS-30 tiene un valor predictivo negativo $(92.6 \%)$ por lo que permite identificar a personas sin depresión (35), y así indagar en los casos de personas que tienen posible depresión.

Una de las principales fortalezas de este estudio es que es uno de los primeros realizados en Centroamérica que examinan la asociación entre desempeño cognitivo y depresión en adultos mayores con deterioro cognitivo leve y con cognición normal. Previamente, PARI realizó un estudio midiendo la coocurrencia entre depresión, deterioro cognitivo y otras variables, planteando factores de riesgo que están asociados a la prevalencia de depresión y DCL (6). La presente investigación profundiza los esfuerzos de PARI, buscando analizar más de cerca la asociación que existe entre depresión y desempeño cognitivo, en grupos diagnósticos. Con nuestros resultados podemos observar cómo la depresión afecta mayormente a sujetos con DCL, en comparación con sujetos con cognición normal, lo que nos acerca más a continuar estudiando el vínculo entre depresión y cognición, y sus posibles repercusiones en los adultos mayores panameños. 


\section{Conclusión}

En esta investigación se estudió la relación entre el desempeño neuropsicológico y la presencia de posible depresión en panameños mayores de 65 años, con cognición normal y DCL. Los resultados demostraron que existe una asociación entre menor desempeño en el dominio de cognición global, en personas con DCL y depresión, en comparación con sujetos con solamente DCL, o cognición normal con depresión. Al mismo tiempo mayor cantidad de síntomas depresivos estuvo asociado con peor rendimiento en los dominios cognitivos de funciones ejecutivas y atención. Nuestro estudio demuestra que existe una relación entre depresión y cognición, cuya dinámica debe continuar siendo estudiada, para encontrar herramientas que brinden diagnóstico temprano y tratamiento a adultos mayores.

\section{Agradecimiento}

Los autores agradecen el apoyo financiero del Sistema Nacional de Investigación (SNI), de la Secretaría Nacional de Ciencia, Tecnología e Innovación (SENACYT). Agradecen también a la Universidad Santa María La Antigua (USMA) y al Instituto de Investigaciones Científicas y Servicios de Alta Tecnología (INDICASAT).

\section{Referencias}

1. United Nations. World Population Prospects 2019: Highlights. A: Futuribles (Paris, France: 1981) [en línia]. 2019, núm. 141, p. 1-39. ISSN 0337-307X. Disponible a: http://creativecommons.org/licenses/by/3.0/igo/.

2. World Bank. Population Pyramids of the World from 1950 to 2100. A: . 2019.

3. Organización Mundial de la Salud. Las 10 principales causas de defunción. A: [en línia]. 2017. Disponible a: http://www.who.int/mediacentre/factsheets/fs310/es/index1.html.

4. American Psychiatric Association. Guía de Consulta de los Criterios de Diagnóstico del DSM-V. 2013. ISBN 9780890425510.

5. Aziz, R. i Steffens, D.C. What are the causes of late-life depression? A: Psychiatric Clinics of North America. 2013, Vol. 36, núm. 4, p. 497-516. ISSN 0193953X. DOI 10.1016/j.psc.2013.08.001.

6. Mourao, R.J. et al. Depressive symptoms increase the risk of progression to dementia in subjects with mild cognitive impairment: systematic review and meta-analysis. A: International Journal of Geriatric Psychiatry. 2016, Vol. 31, núm. 8, p. 905-911. ISSN 10991166. DOI 10.1002/gps.4406.

7. Alzheimer's Association. 2017 Alzheimer's disease facts and figures. A: Alsheimer's Dement. 2017.

8. Liu, Y.C. et al. Depression and dementia in old-old population: History of depression may be associated with dementia onset. the tome project. A: Frontiers in Aging Neuroscience. 2017, Vol. 9, núm. OCT. ISSN 16634365. DOI 10.3389/fnagi.2017.00335.

9. Lee, G.J. et al. Depressive symptoms in mild cognitive impairment predict greater atrophy in alzheimer's disease-related regions. A: Biological Psychiatry. 2012, Vol. 71, núm. 9, p. 814-821. ISSN 00063223. DOI 10.1016/j.biopsych.2011.12.024. 
10. Fritze, F. et al. Depression in mild dementia: Associations with diagnosis, APOE genotype and clinical features. A: International Journal of Geriatric Psychiatry. 2011, Vol. 26, núm. 10, p. 1054-1061. ISSN 08856230. DOI 10.1002/gps.2643.

11. Ismail, Z. et al. Prevalence of depression in patients with mild cognitive impairment. A: $J A M A$ Psychiatry [en línia]. 2017, Vol. 74, núm. 1, p. 58. ISSN 2168-622X. DOI

10.1001/jamapsychiatry.2016.3162. Disponible a:

http:/ / archpsyc.jamanetwork.com/article.aspx?doi=10.1001/jamapsychiatry.2016.3162.

12. Ortiz, G.G. et al. Prevalence of cognitive impairment and depression among a population aged over 60 years in the metropolitan area of Guadalajara, Mexico. A: Current Gerontology and Geriatric Research. 2012, Vol. 2012, p. 1-7. DOI 10.1155/2012/175019.

13. Hudon, C., Belleville, S. i Gauthier, S. The association between depressive and cognitive symptoms in amnestic mild cognitive impairment. A: International Psychogeriatrics. 2008, Vol. 20, núm. 4, p. 710-723. ISSN 10416102. DOI 10.1017/S1041610208007114.

14. Johnson, L.A. et al. Cognitive differences among depressed and non-depressed MCI participants : a project FRONTIER study. A: International Journal of Geriatric Psychiatry. 2013, p. 377382. DOI 10.1002/gps.3835.

15. Villarreal, A.E. et al. Cognitive impairment, depression, and cooccurrence of both among the elderly in panama: differential associations with multimorbidity and functional limitations. A: BioMed Research International. 2016, Vol. 2016, p. 7 pages. DOI 10.1155/2015/718701.

16. Villarreal, A.E. et al. The Panama aging research initiative longitudinal study. A: MEDICC Review [en línia]. 2019. Disponible a: http://mediccreview.org/the-panama-aging-research-initiativelongitudinal-study/.

17. Reisberg, B. et al. Global Deterioration Scale (GDS). A: Psychopharmacology bulletin. 1988, Vol. 24, núm. 4, p. 661-3. ISSN 0048-5764.

18. Folstein, M.F., Folstein, S.E. i McHugh, P.R. «Mini-mental state». A practical method for grading the cognitive state of patients for the clinician. A: Journal of psychiatric research. 1975, Vol. 12, núm. 3 , p. 189-98. ISSN 0022-3956.

19. Albert, M.S. et al. The diagnosis of mild cognitive impairment due to Alzheimer's disease:

Recommendations from the National Institute on Aging-Alzheimer's Association workgroups on. A: Alzheimer's \& Dementia [en línia]. 2011, Vol. 7, núm. 3, p. 270-279. ISSN 1552-5279. DOI 10.1016/j.jalz.2011.03.008.The. Disponible a: http://www.sciencedirect.com/science/article/pii/S155252601100104X.

20. Pfeffer, R. et al. Measurement of functional activities in older adults in the community. A: Journal of Gerontology. 1982, Vol. 37, núm. 3, p. 323-9.

21. Lawton, P. i Brody, E. Assessment of older people: Self-maintaining and instrumental activities of saily living. A: Gerontologist. 1969, Vol. 9, p. 179-186.

22. Yesavage, J.A. et al. Development and validation of a geriatric depression screening scale: a preliminary report. A: Journal of psychiatric research. 1982, Vol. 17, núm. 1, p. 37-49. ISSN 0022-3956.

23. Benedict, R., Schretlen, D. i Groninger, L. Hopkins Verbal Learning Test - Revised: normative data and analysis of inter-form and test-retest reliability. A: The Clinical Neuropsychologist. 1998, Vol. 12, p. 43-55. 
24. Wechsler, D. Wechsler Memory Scale IV. Barcelona: Pearson, 2013.

25. Reitan, R. Validity of the Trail Making Test as an Indicator of Organic Brain Damage. A: Southern Universities Press. 1958, Vol. 8, p. 271-276.

26. Freedman, M. et al. Clock Drawing. 1994. ISBN 9780195059069.

27. Mitchell, A.J. A meta-analysis of the accuracy of the mini-mental state examination in the detection of dementia and mild cognitive impairment. A: Journal of psychiatric research [en línia]. England: 2009, Vol. 43, núm. 4, p. 411-431. ISSN 0022-3956. DOI 10.1016/j.jpsychires.2008.04.014. Disponible a: https://www.ncbi.nlm.nih.gov/pubmed/18579155.

28. Larner, A.J. Cognitive screening instruments for the diagnosis of mild cognitive impairment. A: Progress in Neurology and Psychiatry. 2016, Vol. 20. ISSN 1980-5764. DOI 10.1590/s1980$57642014 \operatorname{dn} 84000010$.

29. Cacho, J. et al. Does the combination of the MMSE and clock drawing test (mini-clock) improve the detection of mild Alzheimer's disease and mild cognitive impairment? A: Journal of Alzheimer's disease: JAD. Netherlands: 2010, Vol. 22, núm. 3, p. 889-896. ISSN 1875-8908 (Electronic). DOI 10.3233/JAD-2010-101182.

30. Cipriani, G. et al. Depression and dementia. A review. A: European Geriatric Medicine [en línia]. Elsevier Masson SAS, 2015, Vol. 6, núm. 5, p. 479-486. ISSN 18787649. DOI 10.1136/bmj.c36. Disponible a: https://www.scopus.com/inward/record.uri?eid=2-s2.0-

84942582212\&doi=10.1136\%2Fbmj.c36\&partnerID=40\&md5=e9af6ced55c021122348c5534fcfeff 0.

31. Saczynski, J.S. et al. Depressive symptoms and risk of dementia: The framingham heart study. A: Neurology. 2010, Vol. 75, núm. 1, p. 35-41. ISSN 00283878. DOI 10.1212/WNL.0b013e3181e62138.

32. Köhler, S. et al. The pattern and course of cognitive impairment in late-life depression. A: Psychological Medicine [en línia]. Cambridge University Press, 2010, Vol. 40, núm. 4, p. 591-602. ISSN 0033-2917. DOI DOI: 10.1017/S0033291709990833. Disponible a:

https://www.cambridge.org/core/article/pattern-and-course-of-cognitive-impairment-in-latelifedepression/60F32B5A81827272C5E042BE3EA0020E.

33. De Assis Faria, C. et al. Cognitive deficits in older adults with mild cognitive impairment in a. A: Dement Neuropsychol. 2018, Vol. 12, núm. 1, p. 19-27. DOI 10.1590/1980-57642018dn12-01000.

34. Nabbe, P. et al. Which DSM validated tools for diagnosing depression are usable in primary care research? A systematic literature review. A: European Psychiatry. 2017, Vol. 39, p. 99-105. ISSN 17783585. DOI 10.1016/j.eurpsy.2016.08.004.

35. Lezcano, H. et al. UTILIDAD DE LA ESCALA DE DEPRESIÓN GERIÁTRICA EN ADULTOS MAYORES ATENDIDOS EN EL SERVICIO DE GERIATRÍA DE LA CAJA DE SEGURO. A: Revista Médico Científica. 2017, Vol. 30, p. 4-10. 